\title{
Hsa_circ_0076305 induces migration-proliferation dichotomy in gastric cancer
}

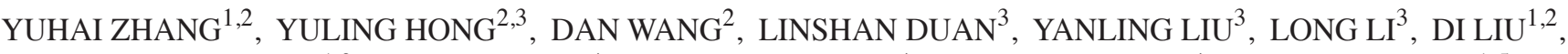 \\ KUNBIN ZHUANG ${ }^{1,2}, \mathrm{CHAOXIN} \mathrm{WEI}^{4}$, GUOGENG ZHENG $^{4}, \mathrm{CHUNYONG} \mathrm{HUO}^{4}$ and GUOYAN LIU ${ }^{1-5}$ \\ ${ }^{1}$ Department of Gastrointestinal Surgery, Zhongshan Hospital of Xiamen University, Xiamen, Fujian 361004; \\ ${ }^{2}$ Department of Basic Medicine, Medical College of Xiamen University, Xiamen, Fujian 361000; \\ ${ }^{3}$ Department of Cancer Prevention and Rehabilitation, Huayan Science and Technology Cancer Prevention and \\ Rehabilitation Research Center, School of Pharmaceutical Sciences Xiamen University, Xiamen, Fujian \\ 361102; ${ }^{4}$ School of Clinical Medicine, Fujian Medical University, Fuzhou, Fujian 350000; ${ }^{5}$ College \\ of Food an Biological Engineering, Jimei University, Xiamen, Fujian 361021, P.R. China
}

Received February 27, 2020; Accepted November 13, 2020

DOI: $10.3892 / \mathrm{ol} .2021 .12481$

\begin{abstract}
Recent studies have demonstrated that circular RNAs (circRNAs) play an important role in the development of gastric cancer (GC). The present study aimed to investigate the role of hsa_circ_0076305 (circPGC) in GC. The levels of circRNAs and mRNAs in AGS cell lines were detected via reverse transcription-quantitative PCR, and western blotting was performed to detect protein expression levels. Functional studies were explored by CCK 8 assay and cell migration assay. Functional studies have indicated that circPGC orchestrates two cellular processes; it inhibits proliferation, and promotes migration and invasion in the GC AGS cell line, a phenomenon called 'migration-proliferation dichotomy', as well as epithelial-to-mesenchymal transition in AGS cells. In addition, circPGC degrades the extracellular matrix and basement membrane through matrix metallopeptidase (MMP)9 and MMP14, providing a microenvironment that facilitates cell migration. The results also demonstrated that circPGC expression is lower in clinical patients with later stages of GC, which is associated with poor prognosis. Taken together, these results suggest that circPGC exhibits migration-proliferation dichotomy during GC development, invasion and migration.
\end{abstract}

\section{Introduction}

Gastric cancer (GC) is one of the most common gastrointestinal malignant tumors, with high morbidity and mortality rates $(1,2)$. In 2015 , China reported $\sim 498,000$

Correspondence to: Dr Guoyan Liu, Department of Gastrointestinal Surgery, Zhongshan Hospital of Xiamen University, 201-209 Hubin South Road, Xiamen, Fujian 361004, P.R. China

E-mail: liuguoyan@xmu.edu.cn

Key words: gastric cancer, cell proliferation, invasion, EMT, migration-proliferation dichotomy mortalities from GC, accounting for $17.7 \%$ of all malignant cases (498,000/2.814 million), and it ranked second among all types of tumors (1). Surgery remains the most effective treatment for GC $(3,4)$. However, identification of important molecular functions in the progression of GC will contribute to the development of novel effective treatment strategies.

Circular RNAs (circRNAs) are a type of closed circular RNA molecule formed by the cyclization of linear RNA sequences at the $5^{\prime}$ and $3^{\prime}$ downstream ends, which lack a 5' cap and 3' poly (adenylate) tail structure (5). CircRNAs are stable in nature and are not easily degraded by nucleic acid endonuclease $(6,7)$. CircRNAs exist in a variety of mammalian cells and are involved in gene transcription and post-transcriptional expression regulation (7). Recently, several studies have demonstrated that circRNAs play an important role in the occurrence and development of different types of diseases $(5,6,8-10)$, including cancer $(11,12)$. In addition, previous studies have reported that the abundance of total circRNAs in cancer tissues is associated with the degree of cancer metastasis $(12,13)$. CircRNAs are abundant in GC and play an important role in the occurrence and development of GC (14-16). Thus, the different expression levels of circRNAs and their molecular mechanisms can provide novel insight and methods for the diagnosis, treatment plan and prognosis of patients with GC.

In the present study, bioinformatics analysis was performed to select an circRNA molecule in GC from the Gene Expression Omnibus (GEO) database (https://www.ncbi.nlm. nih.gov/geo). The results demonstrated that hsa_circ_0076305 (circPGC) was downregulated in GC, suggesting that circPGC may inhibit the proliferation, and promote the migration and invasion of AGS cells. In addition, the clinicopathological characteristics of patients with GC were assessed to identify the migration-proliferation dichotomy role of circPGC in $\mathrm{GC}$ development. The results of the present study demonstrated that the novel circRNA, circPGC, affected the progression of GC with migration-proliferation dichotomy. 


\section{Materials and methods}

Patient samples and cell lines. Bioinformatics analysis was performed to select an circRNA molecule in GC from the GEO database (GSE100170 dataset) (13). A total of nine pairs of GC and paracancerous tissue samples (Table SIII) were collected from the Zhongshan Hospital of Xiamen University between September 2012 and April 2014 and stored at $-80^{\circ} \mathrm{C}$ until further experimentation. In addition, $30 \mathrm{cDNA}$ GC and paracancerous tissue samples were purchased from Shanghai Outdo Biotech Co., Ltd. (cat. no. cDNA-HStmA060CS0).

The AGS GC cell line was kindly provided by the Shanghai Stem Cell Bank of the Chinese Academy of Sciences (https://www.cellbank.org.cn). Cells were maintained in RPMI-1640 medium (Gibco; Thermo Fisher Scientific, Inc.) supplemented with $10 \%$ fetal bovine serum (FBS; Gibco; Thermo Fisher Scientific, Inc.), at $37^{\circ} \mathrm{C}$ with $5 \% \mathrm{CO}_{2}$.

Reverse transcription-quantitative $(R T-q) P C R$. Total RNA was extracted from GC and paracanceroustissues, as well as treated AGS GC cells using TRIzol ${ }^{\circledR}$ reagent (Takara Bio, Inc.). Total RNA was reverse transcribed into cDNA using the PrimeScript RT reagent kit (cat. no. RR047A; Takara Bio, Inc.). qPCR was subsequently performed on an ABI 7500 system using ChamQ SYBR qPCR Master Mix (cat. no. Q311; Vazyme Biotech Co., Ltd.), according to the manufacturer's instructions. The primer sequences used for qPCR are listed in Table SII. Relative expression levels were normalized to the internal reference gene GAPDH. Amplification and detection were run in ABI 7500 system with an initial cycle of $95^{\circ} \mathrm{C}$ for $10 \mathrm{sec}$, followed by 40 cycles of $95^{\circ} \mathrm{C}$ for $5 \mathrm{sec}, 60^{\circ} \mathrm{C}$ for $30 \mathrm{sec}$ and $72^{\circ} \mathrm{C}$ for $5 \mathrm{sec}$. Relative expression levels were calculated using the threshold cycle $\left(2^{-\Delta \Delta \mathrm{Cq}}\right)$ method and normalized to GAPDH $(17,18)$. The primer sequences used are listed in Table SI.

Plasmid construction and transfection. The circPGC cDNA template was extracted from AGS cells and amplified using Prime STAR HS DNA Polymerase (cat. no. R010A; Takara Bio, Inc.). Similarly, the no-load plasmid pcDNA3.1(+) CircRNA Mini Vector which was purchased from the nonprofit plasmid repository Addgene (cat. no. 60648) was linearized via PCR. The target DNA template was extracted from AGS cells. PrimeSTAR ${ }^{\circledR} \mathrm{HS}$ DNA Polymerase was purchased from Taraka (cat. no. R010A). The PCR condition was 1 cycle of $98^{\circ} \mathrm{C}$ for $2 \mathrm{~min}$, and 40 cycles of $98^{\circ} \mathrm{C}$ for $10 \mathrm{sec}, 57^{\circ} \mathrm{C}$ for $5 \mathrm{sec}, 72^{\circ} \mathrm{C}$ for $1 \mathrm{~min}$, and then $1 \mathrm{cycle}$ of $72^{\circ} \mathrm{C}$ for $10 \mathrm{~min}$ followed by cooling down to $4^{\circ} \mathrm{C}$. The PCR products were visualized on SYBR green stained $1 \%$ agarose gels. The primer sequences are listed in Table SI. The results demonstrated that circPGC circularized with exons 3-9 of PGC mRNA (Fig. S1). The fragment of PGC exons 3-9 was constructed into the pcDNA3.1(+) CircRNA Mini Vector via seamless cloning, and subsequently circularized into a circular RNA molecular structure via back splicing (19). The primer sequences used are listed in Table SI. Agarose gel electrophoresis was subsequently performed to separate and purify the DNA fragment using the OMEGA Gel Extraction kit (cat. no. D2500; Omega Bio-Tek, Inc.). The HB-infusion Master mix (Hanbio Biotechnology Co., Ltd.) was used to construct the circPGC expression vector, according to the manufacturer's instructions.

AGS cells $\left(5 \times 10^{6}\right)$ were cultured in RPMI-1640 medium for $24 \mathrm{~h}$ at $37^{\circ} \mathrm{C}$ until they reached $70-80 \%$ confluence.
Cells were transfected with $1 \mu \mathrm{g}$ circPGC expression vector (circPGC) or empty vector (pcDNA 3.1) for $48 \mathrm{~h}$ at $37^{\circ} \mathrm{C}$ using Lipofectamine ${ }^{\circledR} 3000$ reagent (Thermo Fisher Scientific, Inc.) according to the manufacturer's instructions. RT-qPCR analysis was performed to identify the cyclization of circPGC and validate the resistance of circPGC to RNase $\mathrm{R}$ digestion in AGS cells $48 \mathrm{~h}$ post-transfection.

Cell proliferation analysis. The effects of circPGC overexpression on cell proliferation were assessed via the Cell Counting Kit-8 (CCK-8) and colony formation assays. Briefly, AGS cells with stable circPGC overexpression in the logarithmic phase were seeded into 6-well plates at a density of 500 cells/well and incubated for 10 days at $37^{\circ} \mathrm{C}$ in a $5 \% \mathrm{CO}_{2}$ incubator. Following incubation, cells were fixed with $4 \%$ paraformaldehyde for $20 \mathrm{~min}$ and stained with $0.1 \%$ crystal violet for $2 \mathrm{~min}$ at room temperature. Cell colonies were observed and counted under an inverted fluorescence microscope (Olympus IX51; Olympus Corporation; magnification, x200).

Similarly, the CCK-8 assay (Beyotime Institute of Biotechnology) was performed according to the manufacturer's instructions. AGS cells (3,000 cells/well) were incubated with CCK-8 reagent for $3 \mathrm{~h}$ and cell proliferation was subsequently analyzed at a wavelength of $450 \mathrm{~nm}$.

Wound healing assay. Cells were transfected with plasmid and cultured until they reached $100 \%$ confluence. A wound gap was carefully scratched across the surface of the cells using a sterile $10 \mu \mathrm{l}$ pipette tip, and the scraped cells were removed using PBS. Cells were re-cultured in serum-free RPMI-1640 medium and incubated at $37^{\circ} \mathrm{C}$ in a $5 \% \mathrm{CO}_{2}$ incubator. Cells were observed under an inverted fluorescence microscope (magnification, x200, Olympus IX51; Olympus Corporation).

Cell migration and invasion assays. A 24-well chamber with an $8-\mu \mathrm{m}$ pore size (cat. no. 3422; Corning, Inc) was used to detect the cell migratory and invasive abilities. A total of $5 \times 10^{4}$ AGS cells were plated in the upper chambers of Transwell plates in $200 \mu \mathrm{l}$ serum-free medium. For the invasion assay, Transwell membranes were precoated with Matrigel in $4^{\circ} \mathrm{C}$ for $12 \mathrm{~h}$ (BD Biosciences). Medium supplemented with 10\% FBS was plated in the lower chambers. Following incubation at $37^{\circ} \mathrm{C}$ for $12 \mathrm{~h}$, cells on the upper chambers were removed using a cotton swab, while the migratory cells were fixed with $4 \%$ paraformaldehyde for $30 \mathrm{~min}$ and stained with $0.1 \%$ crystal violet for $20 \mathrm{~min}$ at room temperature. Stained cells were counted using an inverted fluorescence microscope (magnification, x200).

Western blotting. Total cellular protein was extracted using RIPA lysis buffer (cat. no. 20101ES60; Yeasen Biotech Shanghai) and quantified using a BCA kit (cat. no. 23252; Thermo Fisher Scientific, Inc.). Protein samples $(50 \mu \mathrm{g})$ were loaded and separated by $12 \%$ SDS-PAGE. The separated proteins were subsequently transferred onto polyvinylidene difluoride membranes (EMD Millipore) and blocked with 5\% non-fat milk for $1 \mathrm{~h}$ at room temperature. The membranes were incubated with primary antibodies against: E-cadherin (dilution ratio 1:2,000; cat. no. SC7870; Santa Cruz Biotechnology, Inc.), N-cadherin (dilution ratio 1:1,000; cat. no. 22018-1; ProteinTech Group, Inc.), Snail (dilution ratio 1:2,000; cat. no. 3879; Cell Signaling 


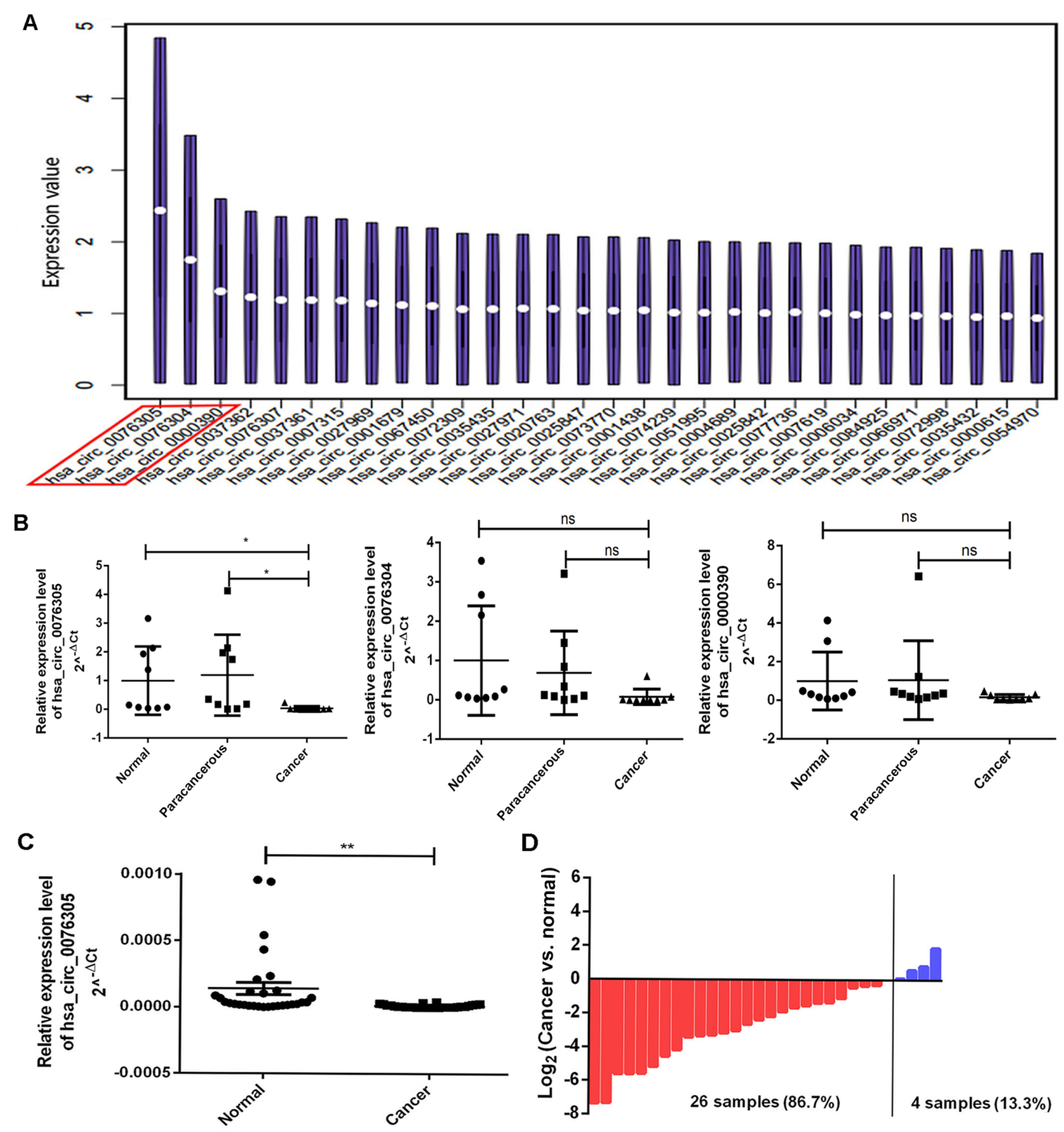

Figure 1. CircPGC is downregulated in GC. (A) The first 30 circular RNAs with significantly downregulated expression in GC tissues. (B) CircPGC expression was significantly downregulated in GC tissues $(\mathrm{n}=9)$ compared with normal and paracancerous tissue samples, while hsa_circ_0076304 and hsa_circ_0000390 expression levels were not significantly downregulated in GC tissues. (C) CircPGC expression was significantly downregulated in GC cDNA samples (n=30), (D) CircPGC expression was downregulated in 26 samples (86.7\%) and only upregulated in 4 samples (13.3\%). Data are presented as the mean \pm standard error of the mean. ${ }^{*} \mathrm{P}<0.05,{ }^{* *} \mathrm{P}<0.01$. CircPGC, hsa_circ_0076305; GC, gastric cancer; ns, not statistically significant.

Technology, Inc.), Twist (dilution ratio 1:2,000; cat. no. ab49254; Abcam), Vimentin (dilution ratio 1:1,000; cat. no. 10366-1; ProteinTech Group, Inc.), $\beta$-catenin (dilution ratio 1:2,000; cat. no. 8480; Cell Signaling Technology, Inc.), matrix metallopeptidase (MMP)14 (dilution ratio 1:2,000; cat. no. MAB3328; Merck KGaA), MMP9 (dilution ratio 1:2,000; cat. no. ab38898; Abcam), Collagen I (dilution ratio 1:1,000; cat. no. ab138492; Abcam), proliferating cell nuclear antigen (PCNA; dilution ratio 1:1,000; cat. no. 60097; ProteinTech Group, Inc.), myc proto-oncogene protein (c-Myc; dilution ratio 1:2,000; cat. no. 1472-1; Abcam), epidermal growth factor receptor (EGFR; dilution ratio 1:2,000; cat. no. 4267; Cell Signaling Technology, Inc.) and $\beta$-actin (dilution ratio 1:5,000; cat. no. 60008-1; ProteinTech Group, Inc.), overnight at $4^{\circ} \mathrm{C}$. Membranes were washed twice with PBS and subsequently incubated with secondary antibodies (dilution ratio 1:5,000; cat. nos. G21040 and G21234; Thermo Fisher Scientific, Inc.) conjugated to horseradish peroxidase (Merck KGaA) for $1 \mathrm{~h}$ at room temperature. Protein bands were visualized using enhanced chemiluminescent reagents (Pierce; Thermo Fisher Scientific, Inc.).

Statistical analysis. GraphPad Prism 6.0 software (GraphPad Software, Inc.) was used to perform statistical analysis. All experiments were performed in duplicate and repeated at least three times and data are presented as the mean \pm standard error of the mean SEM. Unpaired Student's t-test was used to compare differences between two groups. The association between circPGC expression and the clinicopathological characteristics of patients with GC was assessed using Pearson's correlation and tumor-node-metastasis (TNM) system analysis (20). $\mathrm{P}<0.05$ was considered to indicate a statistically significant difference.

\section{Results}

circPGC expression is downregulated in GC. According to the GEO database, using the non-coding RNA GSE100170 
A

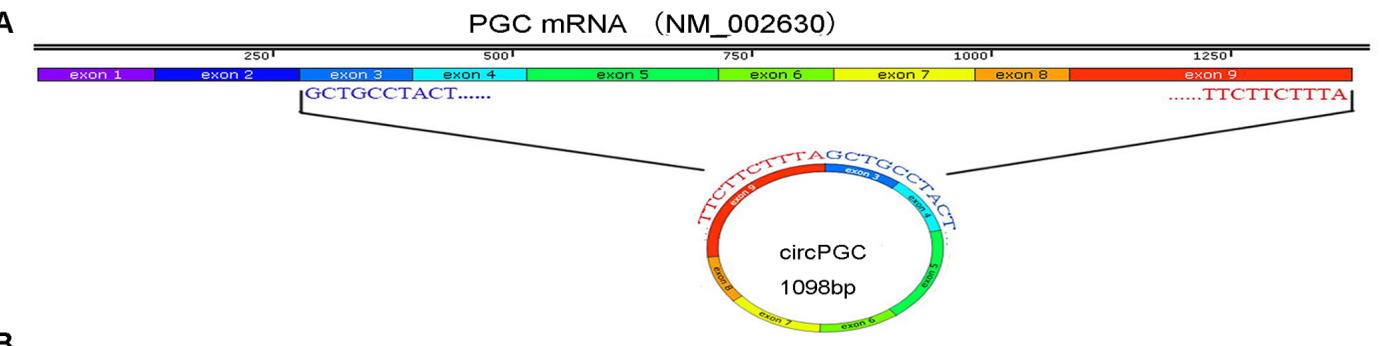

B
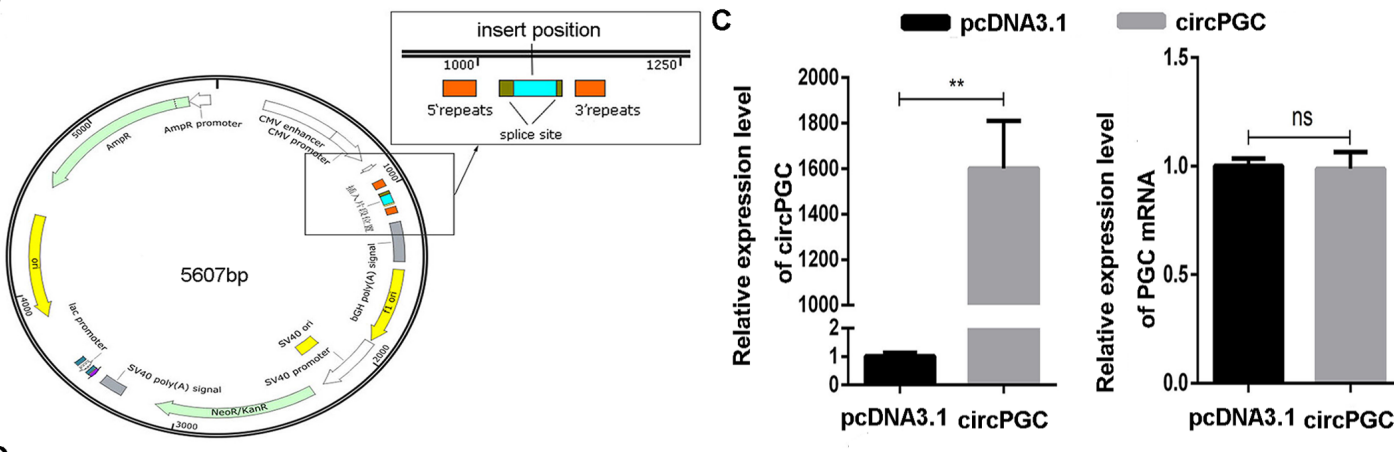

D

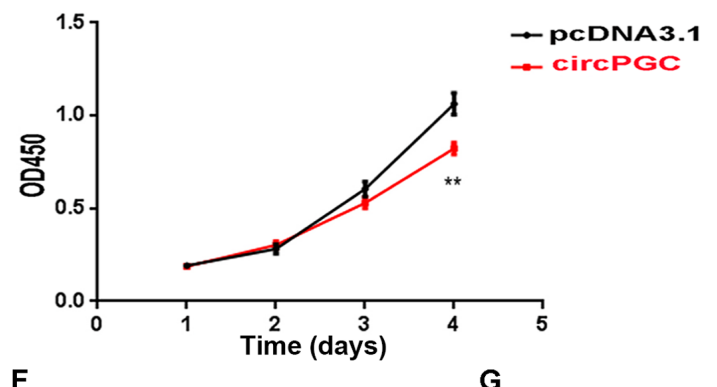

$\mathbf{F}$

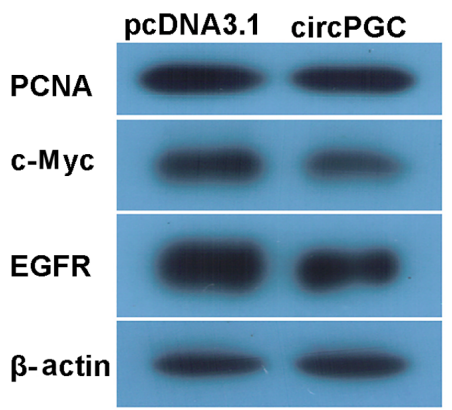

G

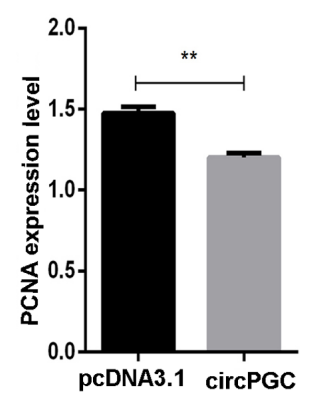

E

pcDNA3.1
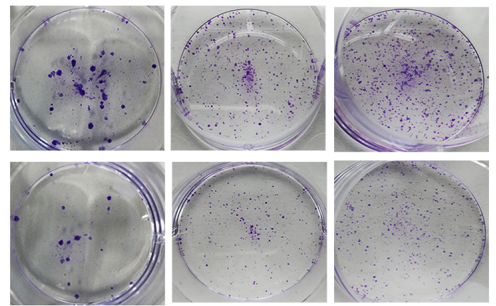

circPGC
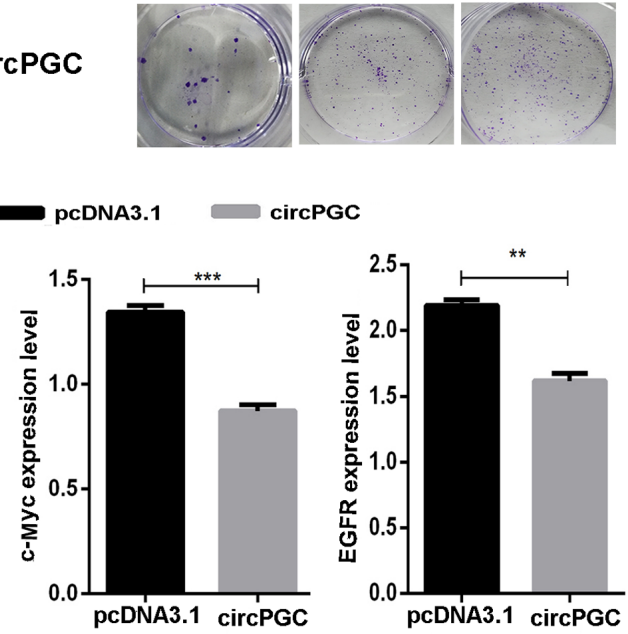

Figure 2. Overexpression of circPGC inhibits AGS cell proliferation. (A) CircPGC circularized with exon 3-9 of PGC mRNA. (B) The fragment of PGC exon 3-9 was constructed into the pcDNA3.1(+) CircRNA Mini Vector via seamless cloning. (C) Following transfection with circPGC for 48 h, circPGC and PGC mRNA expression levels were detected via reverse transcription-quantitative PCR analysis. (D) Cell Counting Kit-8 assay was performed to assess the effect of circPGC on AGS cell proliferation. (E) The results of the colony formation assay demonstrated that circPGC inhibited the colony formation ability of AGS cells. (F) Western blot analysis demonstrated that overexpression of circPGC inhibited the expression levels of c-Myc, EGFR and PCNA. (G) Western blot quantitative results of PCNA, c-Myc and EGFR. Data are presented as the mean \pm standard error of the mean. ${ }^{* *} \mathrm{P}<0.01,{ }^{* * * *} \mathrm{P}<0.001 . \mathrm{CircPGC}$, hsa_circ_0076305; EGFR, epidermal growth factor receptor; PCNA, proliferating cell nuclear antigen; ns, not statistically significant; OD, optical density.

dataset of GC and paracancerous tissue samples, the study (13) demonstrated that a significant difference was observed in 713 circRNAs between GC and paracancerous tissues. Among these, three circRNAs with significant differences (circPGC, hsa_circ_0076304 and hsa_circ_0000390) were selected for further experimentation (Fig. 1A).

To determine whether circPGC, hsa_circ_0076304 and hsa_circ_0000390 were downregulated in GC, the expression of these circRNAs was assessed in nine pairs of GC and paracancerous tissue samples. The results demonstrated that only circPGC was significantly downregulated in GC $(\mathrm{P}<0.05$; Fig. 1B). Furthermore, circPGC expression was assessed in 30 pairs of GC and paracancerous tissue samples and the results demonstrated that circPGC expression was significantly downregulated in GC $(\mathrm{P}<0.01$; Fig. $1 \mathrm{C})$. Among the 30 pairs of tissue samples, circPGC expression was downregulated in 26 patients $(86.7 \%)$ and only upregulated in 4 patients (13.3\%) (Fig. 1D).

Overexpression of circPGC inhibits AGS cell proliferation. The results demonstrated that circPGC circularized with exons 3-9 of PGC mRNA (Fig. 2A). To study the function of circPGC in GC, the fragment of PGC exons 3-9 was constructed into the pcDNA3.1(+) CircRNA Mini Vector via 
seamless cloning (Fig. 2B). Following transient transfection of the circPGC expression vector into AGS cells for $48 \mathrm{~h}$, transfection efficiency was detected via RT-qPCR analysis. The results demonstrated that circPGC expression significantly increased in AGS cells following transfection $(\mathrm{P}<0.01$; Fig. 2C). RT-qPCR analysis was also performed to detect PGC mRNA expression following transfection with circPGC, and the results demonstrated that PGC mRNA expression was not affected by circPGC ( $\mathrm{P}>0.05$; Fig. 2C).

To assess whether overexpression of circPGC regulated cell proliferation in $\mathrm{GC}$, the $\mathrm{CCK}-8$ and colony formation assays were performed to determine the role of circPGC in AGS cells. The results of the CCK- 8 assay demonstrated that AGS cell proliferation significantly decreased following overexpression of circPGC $(\mathrm{P}<0.01$; Fig. 2D). Similarly, the results of the colony formation assay demonstrated that circPGC notably inhibited the colony formation ability of AGS cells (Fig. 2E). To determine the molecular mechanism underlying the effect of circPGC on GC, western blot analysis was performed to detect the expression levels of proliferation-related proteins, including c-Myc (21), EGFR (22) and PCNA (23). The results demonstrated that overexpression of circPGC significantly inhibited the expression levels of c-Myc, EGFR and PCNA $(\mathrm{P}<0.05$; Fig. $2 \mathrm{~F}$ and $\mathrm{G})$. Taken together, these results suggest that circPGC inhibits AGS cell proliferation.

Overexpression of circPGC promotes migration and invasion of AGS cells. Tumor infiltration is the process of malignant tumor cells migrating from their original site to surrounding normal tissues, which is closely associated with the prognosis of patients (24). The wound healing assay was performed to assess whether circPGC affects the migratory and invasive abilities of GC cells. The results demonstrated that the wound gap notably narrowed following transfection of AGS cells with circPGC for $48 \mathrm{~h}(\mathrm{P}<0.05$; Fig. 3A). In addition, the results of the migration and invasion assays demonstrated that overexpression of circPGC promoted cell migration and invasion of AGS cells $(\mathrm{P}<0.05$; Fig. 3B). Collectively, these results suggest that circPGC promotes the migratory and invasive abilities of AGS cells.

Epithelial-to-mesenchymal transition (EMT) is a process in which epithelial cells transform into mesenchymal cells (25), and a previous study suggested that EMT is closely associated with tumor migration and invasion (26). During the EMT process, some proteins, including E-cadherin (27), $\beta$-catenin (28), Snail, Twist, Vimentin (29), and N-cadherin regulate the cell infiltration capacity (30). In the present study, western blot analysis demonstrated that overexpression of circPGC significantly downregulated E-cadherin expression and significantly upregulated the expression levels of Vimentin, $\beta$-catenin, Snail and Twist $(\mathrm{P}<0.05)$, while $\mathrm{N}$-cadherin expression $(\mathrm{P}>0.05)$ remained unchanged (Fig. $3 \mathrm{C}$ and $\mathrm{D})$.

Previous studies have reported that the expression levels of MMP9, MMP14 and Collagen I also affect cell migration (31-33). In the present study, western blot analysis demonstrated that the expression levels of MMP9, MMP14 and Collagen I were significantly upregulated following overexpression of circPGC $(\mathrm{P}<0.05 ;$ Fig. $3 \mathrm{C}$ and $\mathrm{E})$. Taken together, these results suggest that circPGC enhances the migratory and invasive abilities of AGS cells.
Association between circPGC expression and clinicopathological characteristics of patients with GC. To understand the role of circPGC in GC, the tumor-node-metastasis (TNM) system (20) was used, which is a basic criterion for the classification of patients with cancer, their prognosis and treatment plan (34), whereby high TNM stages (stage III or IV) indicate a poor prognosis (20).

The association between circPGC expression and the clinicopathological characteristics of patients with GC was assessed using $30 \mathrm{cDNA}$ GC and paracancerous tissue samples. As presented in Table SIII, 4/13 patients (31\%) with a low TNM stage (stage I or II) had high circPGC expression, while $9 / 13$ patients $(69 \%)$ had low circPGC expression. In addition, all patients with a high TNM stage (stage III or IV) had low circPGC expression. The association between circPGC expression and TNM stage suggests that circPGC is associated with poor prognosis.

Patients in the T3 and T4 stages (advanced GC) had low circPGC expression, while those in the T2 stage had high circPGC expression $(\mathrm{P}<0.05 ;$ Fig. 4A). This result suggests that circPGC expression gradually decreases with GC progressions, indicating that low circPGC expression may promote in situ infiltration in GC cells. Similarly, in patients with lymph node metastasis (N1-N3), circPGC expression was demonstrated to gradually decrease; however, no statistically significant differences were observed between the subgroups ( $\mathrm{P}>0.05$; Fig. 4B).

\section{Discussion}

The results of the present study demonstrated the dual function of circPGC in GC progression, one suppressing and the other promoting. To the best of our knowledge, the present study was the first to demonstrate that circPGC regulates cell proliferation and migration dichotomy in GC cells. Overexpression of circPGC promoted cell migration and invasion, and inhibited proliferation of GC cells. Notably, the results of the present study demonstrated that circPGC expression was downregulated in advanced GC, and acted as an independent molecule for poor patient prognosis. From the perspective of advanced GC, it was speculated that low circPGC expression may promote the proliferation of GC cells to affect the prognosis at this stage, instead of promoting metastasis. Taken together, the results of the present study suggest that low circPGC expression may be a candidate for the prognosis of GC.

It is well-known that malignant proliferation and metastasis are two of the dominant characteristics of cancer, which occur simultaneously (35). However, a phenomenon called migration-proliferation dichotomy has demonstrated that cells do not proliferate and migrate simultaneously (36). This dichotomy has reported that some molecules, such as Girdin and EGFR, are key regulators within the growth factor signaling network during cell migration $(37,38)$. A previous study demonstrated that EGFR interacts with E-cadherin to promote proliferation by activating the MAPK pathway (39). Furthermore, patients with tumors bearing specific mutations in EGFR or overexpressing EGFR have a good clinical response to selective EGFR inhibitors $(22,40,41)$. However, in a previous study, when the tumor cells developed resistance to the EGFR inhibitor, some cells exhibited mesenchymal characteristics and low EGFR expression, survived and underwent 

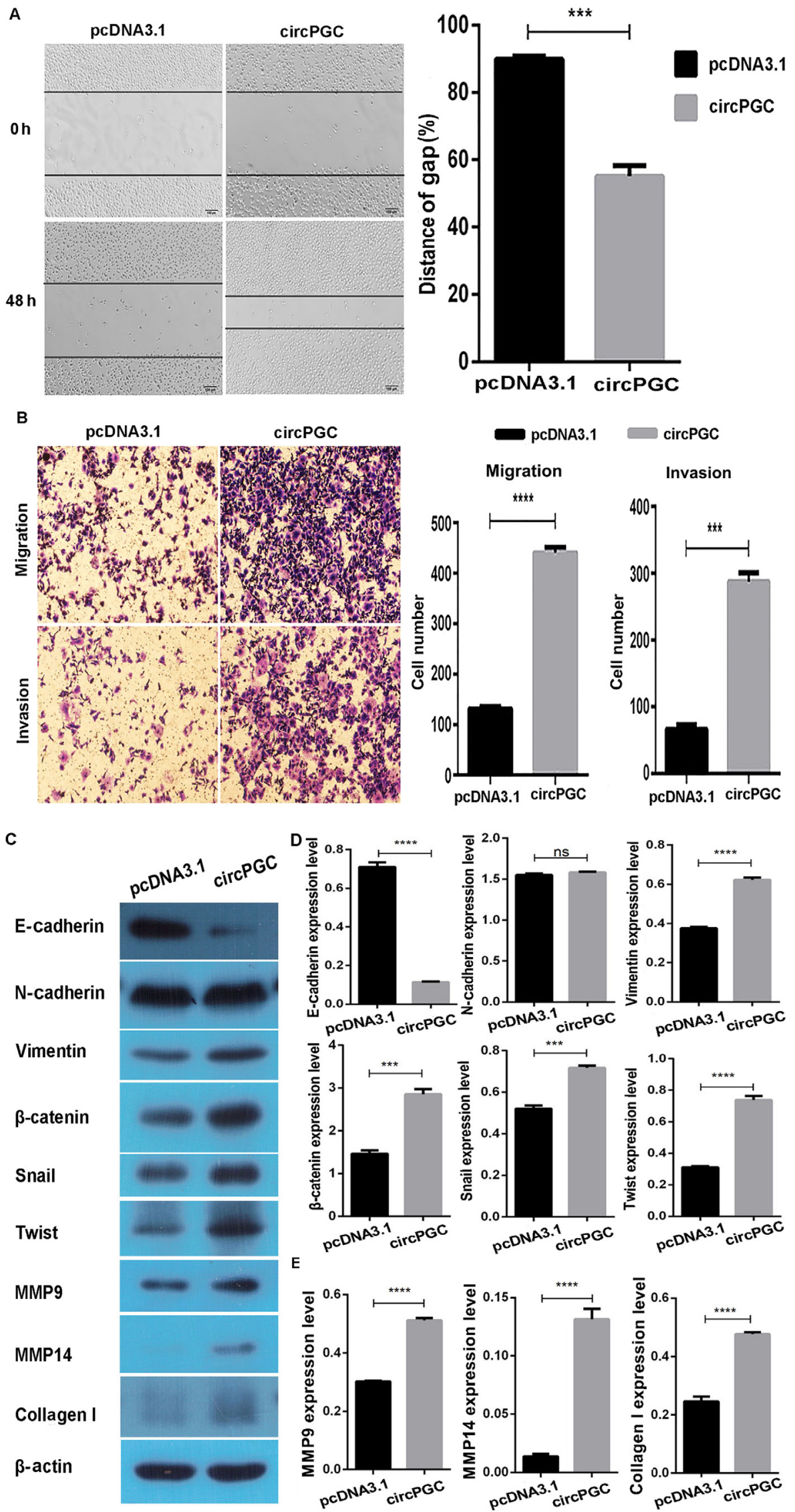

Figure 3. Overexpression of circPGC promotes migration and invasion of AGS cells. (A) The wound healing assay was performed to assess the effect of circPGC on AGS cell migration. (B) The results of the cell migration and invasion assays demonstrated that overexpression of circPGC promoted the cell migratory and invasive abilities of AGS cells. (C) CircPGC was demonstrated to regulate the expression levels of E-cadherin, Vimentin, $\beta$-catenin, Snail, Twist, MMP9, MMP14 and Collagen I. (D) Western blot quantitative results of E-cadherin, Vimentin, $\beta$-catenin, Snail and Twist. (E) Western blot quantitative results of MMP9, MMP14 and Collagen I. Data are presented as the mean \pm standard error of the mean. ${ }^{* * *} \mathrm{P}<0.001,{ }^{* * * * *} \mathrm{P}<0.0001 . \mathrm{CircPGC}, \mathrm{hsa}-\mathrm{circ} \_0076305$; MMP, matrix metallopeptidase; ns, not statistically significant. 
A

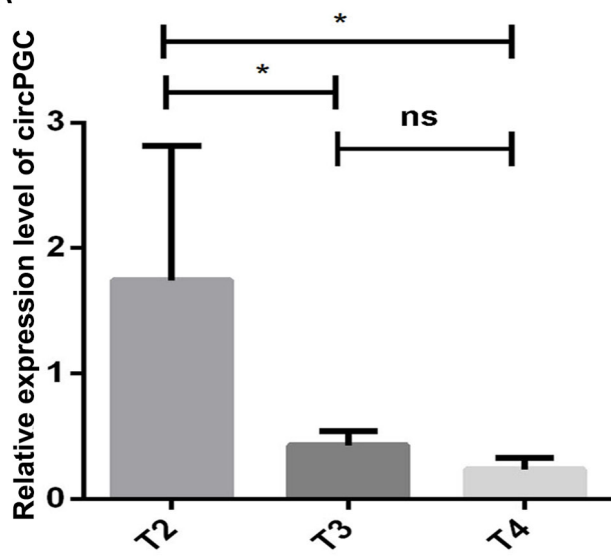

B

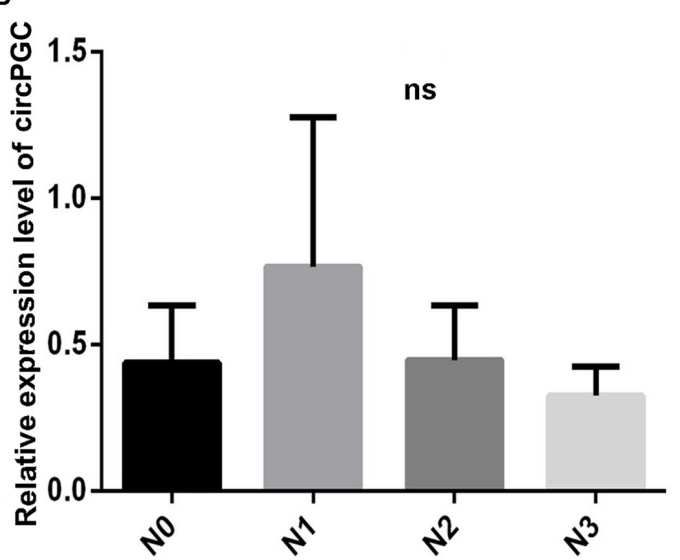

Figure 4. Association between circPGC expression and the clinicopathological characteristics of patients with GC. (A) circPGC expression in patients with advanced GC was assessed by analyzing infiltration depth [T2-T4; 3 (T2), 18 (T3), and 8 (T4)]. (B) In patients with lymph node metastasis [N1-N3; 7 (N1), 8 (N2) and 6 (N3)], circPGC expression gradually decreased; however, no statistically significant differences were observed. Data are presented as the mean \pm standard error of the mean. ${ }^{*} \mathrm{P}<0.05$. CircPGC, hsa_circ_0076305; GC, gastric cancer; ns, not statistically significant.

EMT (25). These findings indicate that EGFR and E-cadherin play a dominant role in migration-proliferation dichotomy. Consistent with previous findings $(22,25,42)$, the results of the present study demonstrated that both EGFR and E-cadherin expression levels decreased following overexpression of circPGC, demonstrating the occurrence of migration-proliferation dichotomy.

Based on the migration-proliferation dichotomy, it was demonstrated that circPGC decreased the expression levels of EGFR, PCNA and c-Myc to suppress cell proliferation. Simultaneously, it was demonstrated that circPGC promoted cell migration by decreasing E-cadherin expression. E-cadherin is a well-characterized cell surface molecule expressed in epithelial cells or certain cancer cells, which plays a major role in cell migration and adhesion $(42,43)$. EMT is a process by which tumor cells lose their epithelial phenotype, tight connections and polarity, and acquire a mesenchymal phenotype, with advanced migratory and invasive abilities $(26,44)$. The results of the present study demonstrated that overexpression of circPGC increased the expression levels of Vimentin, $\beta$-catenin, Snail and Twist, and decreased E-cadherin expression. Notably, circPGC had no effect on $\mathrm{N}$-cadherin expression. A potential explanation for this phenomenon is that overexpression of circPGC may only lead to partial EMT, or there may be other potential molecular mechanisms that influence the effect of circPGC on $\mathrm{N}$-cadherin expression. $\mathrm{ECM}$ is an integral part of the tumor microenvironment that provides structural support to tumor cells $(45,46)$. The results of the present study also demonstrated that overexpression of circPGC degraded ECM and basement membrane to provide a migration environment via Collagen I, MMP9 and MMP14.

The clinicopathological characteristics of 30 patients with GC were also assessed, and the results demonstrated that circPGC may be associated with the prognosis of patients. Notably, patients with low circPGC expression developed advanced GC. Thus, circPGC has the potential to be developed as a novel target for GC prevention and therapy. Although the present study only assessed the dual effects of circPGC on migration and proliferation in AGS cells in vitro, the underlying molecular mechanism of this phenomenon has not yet been determined. In addition, the results presented here require verification through in vivo experiments and investigations using others GC cell lines.

\section{Acknowledgements}

Not applicable.

\section{Funding}

The present study was supported by grants from The National Natural Science Foundation of China (grant no. 81870388) and the Special Project for Marine Economic Development of Xiamen City (grant no. 17GYY001NF01).

\section{Availability of data and materials}

The datasets generated and/or analyzed during the present study are available from the corresponding author upon reasonable request.

\section{Authors' contributions}

YZ and YH designed the present study, performed the experiments, analyzed the data and drafted the initial manuscript. DW, LD, YL and LL interpreted and analyzed the data. DL, KZ, CW, GZ and $\mathrm{CH}$ collected and assembled the clinical data and analyzed the data. YH and GL planned the experiments, revised the manuscript for intellectual content and submitted the manuscript for publication. All authors have read and approved the final version of the manuscript.

\section{Ethics approval and consent to participate}

The present study was approved by the Clinical Research Ethics Committee of the Zhongshan Hospital of Xiamen University, and performed in accordance with IRB-approved institutional protocols. Informed consent was provided by all patients prior to the study start. 


\section{Patient consent for publication}

Not applicable.

\section{Competing interests}

The authors declare that they have no competing interests.

\section{References}

1. Chen W, Zheng R, Baade PD, Zhang S, Zeng H, Bray F, Jemal A, Yu XQ and He J: Cancer statistics in China, 2015. CA Cancer J Clin 66: 115-132, 2016.

2. Bonelli P, Borrelli A, Tuccillo FM, Silvestro L, Palaia R and Buonaguro FM: Precision medicine in gastric cancer. World J Gastrointest Oncol 11: 804-829, 2019.

3. Symeonidis D, Diamantis A, Bompou E and Tepetes K: Current role of lymphadenectomy in gastric cancer surgery. J BUON 24: 1761-1767,2019.

4. Zhang F, Huang X, Song Y, Gao P, Zhou C, Guo Z, Shi J, Wu Z and Wang Z: Conversion surgery for stage IV gastric cancer. Front Oncol 9: 1158, 2019.

5. Shang Q, Yang Z, Jia R and Ge S: The novel roles of circRNAs in human cancer. Mol Cancer 18: 6, 2019.

6. Chen LL and Yang L: Regulation of circRNA biogenesis. RNA Biol 12: 381-388, 2015.

7. Jeck WR, Sorrentino JA, Wang K, Slevin MK, Burd CE, Liu J, Marzluff WF and Sharpless NE: Circular RNAs are abundant, conserved, and associated with ALU repeats. RNA 19: 141-157, 2013

8. Chen X, Yang T, Wang W, Xi W, Zhang T, Li Q, Yang A and Wang T: Circular RNAs in immune responses and immune diseases. Theranostics 9: 588-607, 2019.

9. Rong D, Tang W, Li Z, Zhou J, Shi J, Wang H and Cao H: Novel insights into circular RNAs in clinical application of carcinomas. OncoTargets Ther 10: 2183-2188, 2017.

10. Han B, Chao J and Yao H: Circular RNA and its mechanisms in disease: From the bench to the clinic. Pharmacol Ther 187: 31-44, 2018.

11. Dong Y, He D, Peng Z, Peng W, Shi W, Wang J, Li B, Zhang C and Duan C: Circular RNAs in cancer: An emerging key player. J Hematol Oncol 10: 2, 2017.

12. He J, Xie Q, Xu H, Li J and Li Y: Circular RNAs and cancer Cancer Lett 396: 138-144, 2017.

13. Dang Y, Ouyang X, Zhang F, Wang K, Lin Y, Sun B, Wang Y, Wang L and Huang Q: Circular RNAs expression profiles in human gastric cancer. Sci Rep 7: 9060, 2017.

14. Sui W, Shi Z, Xue W, Ou M, Zhu Y, Chen J, Lin H, Liu F and Dai Y: Circular RNA and gene expression profiles in gastric cancer based on microarray chip technology. Oncol Rep 37: 1804-1814, 2017.

15. Shen Y, Zhang J, Fu Z, Zhang B, Chen M, Ling X and Zou X Gene microarray analysis of the circular RNAs expression profile in human gastric cancer. Oncol Lett 15: 9965-9972, 2018

16. Huang YS, Jie N, Zou KJ and Weng Y: Expression profile of circular RNAs in human gastric cancer tissues. Mol Med Rep 16: 2469-2476, 2017

17. Bustin SA, Benes V, Garson JA, Hellemans J, Huggett J, Kubista M, Mueller R, Nolan T, Pfaffl MW, Shipley GL, et al: The MIQE guidelines: Minimum information for publication of quantitative real-time PCR experiments. Clin Chem 55: 611-622, 2009.

18. Livak KJ and Schmittgen TD: Analysis of relative gene expression data using real-time quantitative PCR and the 2(-Delta Delta C(T)) method. Methods 25: 402-408, 2001.

19. Liang D and Wilusz JE: Short intronic repeat sequences facilitate circular RNA production. Genes Dev 28: 2233-2247, 2014.

20. Sellers AH: The clinical classification of malignant tumours: The TNM system. Can Med Assoc J 105: 836, passim, 1971.

21. Dang CV: MYC on the path to cancer. Cell 149: 22-35, 2012.

22. Mitchell RA, Luwor RB and Burgess AW: Epidermal growth factor receptor: Structure-function informing the design of anticancer therapeutics. Exp Cell Res 371: 1-19, 2018.

23. Juríková M, Danihel L', Polák Š and Varga I: Ki67, PCNA, and MCM proteins: Markers of proliferation in the diagnosis of breast cancer. Acta Histochem 118: 544-552, 2016.

24. Ahn HS, Lee HJ, Hahn S, Kim WH, Lee KU, Sano T, Edge SB and Yang HK: Evaluation of the seventh American Joint Committee on Cancer/International Union Against Cancer Classification of gastric adenocarcinoma in comparison with the sixth classification. Cancer 116: 5592-5598, 2010
25. Tulchinsky E, Demidov O, Kriajevska M, Barlev NA and Imyanitov E: EMT: A mechanism for escape from EGFR-targeted therapy in lung cancer. Biochim Biophys Acta Rev Cancer 1871: 29-39, 2019

26. Nieto MA, Huang RY, Jackson RA and Thiery JP: Emt: 2016 Cell 166: 21-45, 2016

27. Christofori $\mathrm{G}$ and Semb H: The role of the cell-adhesion molecule E-cadherin as a tumour-suppressor gene. Trends Biochem Sci 24 73-76, 1999.

28. Kim K, Lu Z and Hay ED: Direct evidence for a role of beta-catenin/LEF-1 signaling pathway in induction of EMT. Cell Biol Int 26: 463-476, 2002.

29. Mendez MG, Kojima S and Goldman RD: Vimentin induces changes in cell shape, motility, and adhesion during the epithelial to mesenchymal transition. FASEB J 24: 1838-1851, 2010.

30. Hazan RB, Phillips GR, Qiao RF, Norton L and Aaronson SA: Exogenous expression of $\mathrm{N}$-cadherin in breast cancer cells induces cell migration, invasion, and metastasis. J Cell Biol 148 779-790, 2000.

31. Zhang K, Corsa CA, Ponik SM, Prior JL, Piwnica-Worms D, Eliceiri KW, Keely PJ and Longmore GD: The collagen receptor discoidin domain receptor 2 stabilizes SNAIL1 to facilitate breast cancer metastasis. Nat Cell Biol 15: 677-687, 2013.

32. Lin CY, Tsai PH, Kandaswami CC, Lee PP, Huang CJ, Hwang JJ and Lee MT: Matrix metalloproteinase-9 cooperates with transcription factor Snail to induce epithelial-mesenchymal transition. Cancer Sci 102: 815-827, 2011.

33. Yan T, Lin Z, Jiang J, Lu S, Chen M, Que H, He X, Que G, Mao J, Xiao J, et al: MMP14 regulates cell migration and invasion through epithelial-mesenchymal transition in nasopharyngeal carcinoma. Am J Transl Res 7: 950-958, 2015.

34. Amin MB, Greene FL, Edge SB, Compton CC, Gershenwald JE, Brookland RK, Meyer L, Gress DM, Byrd DR and Winchester DP: The Eighth Edition AJCC Cancer Staging Manual: Continuing to build a bridge from a population-based to a more 'personalized' approach to cancer staging. CA Cancer J Clin 67: 93-99, 2017.

35. van Denderen BJ and Thompson EW: Cancer: The to and fro of tumour spread. Nature 493: 487-488, 2013.

36. Ghosh P, Beas AO, Bornheimer SJ, Garcia-Marcos M, Forry EP, Johannson C, Ear J, Jung BH, Cabrera B, Carethers JM, et al: A G\{alpha\}i-GIV molecular complex binds epidermal growth factor receptor and determines whether cells migrate or proliferate. Mol Biol Cell 21: 2338-2354, 2010

37. Ghosh P, Garcia-Marcos M, Bornheimer SJ and Farquhar MG: Activation of Galphai3 triggers cell migration via regulation of GIV. J Cell Biol 182: 381-393, 2008.

38. Beas AO, Taupin V, Teodorof C, Nguyen LT, Garcia-Marcos M and Farquhar MG: Gas promotes EEA1 endosome maturation and shuts down proliferative signaling through interaction with GIV (Girdin). Mol Biol Cell 23: 4623-4634, 2012.

39. Pece S and Gutkind JS: Signaling from E-cadherins to the MAPK pathway by the recruitment and activation of epidermal growth factor receptors upon cell-cell contact formation. J Biol Chem 275: 41227-41233, 2000

40. Moiseyenko VM, Procenko SA, Levchenko EV, Barchuk AS, Moiseyenko FV, Iyevleva AG, Mitiushkina NV, Togo AV, Semionov II, Ivantsov AO, et al: High efficacy of first-line gefitinib in non-Asian patients with EGFR-mutated lung adenocarcinoma. Onkologie 33: 231-238, 2010.

41. Edinger N, Lebendiker M, Klein S, Zigler M, Langut $\mathrm{Y}$ and Levitzki A: Targeting polyIC to EGFR over-expressing cells using a dsRNA binding protein domain tethered to EGF. PLoS One 11: e0162321, 2016

42. Qian X, Karpova T, Sheppard AM, McNally J and Lowy DR: E-cadherin-mediated adhesion inhibits ligand-dependent activation of diverse receptor tyrosine kinases. EMBO J 23: 1739-1748, 2004

43. Chen D, Wu Z, Luo LJ, Huang X, Qian WQ, Wang H, Li SH and Liu J: E-cadherin maintains the activity of neural stem cells and inhibits the migration. Int J Clin Exp Pathol 8: 14247-14251, 2015.

44. Boyer B, Vallés AM and Edme N: Induction and regulation of epithelial-mesenchymal transitions. Biochem Pharmacol 60: 1091-1099, 2000

45. Hynes RO: The extracellular matrix: Not just pretty fibrils. Science 326: 1216-1219, 2009.

46. Venning FA, Wullkopf L and Erler JT: Targeting ECM Disrupts Cancer Progression. Front Oncol 5: 224, 2015.

This work is licensed under a Creative Commons Attribution-NonCommercial-NoDerivatives 4.0 International (CC BY-NC-ND 4.0) License. 